\title{
NUESTROS BECARIOS DESTACADOS
}

"La sabiduría suprema es tener sueños bastante grandes para no perderlos de vista mientras se persiguent". William Faulkner. 


\section{REUNIÓN DE COMUNICACIONES CIENTÍFICAS Y TECNOLÓGICAS DE LA UNNE}

esde 1994 la Universidad Nacional del Nordeste (UNNE) congrega
anualmente, a investigadores y becarios, a un evento de comunica-
ción de sus avances y resultados, con el objetivo de difundir, compartir e interrelacionar las tareas de los distintos grupos de investigación, enfatizando una estrategia institucional de pertinencia y transferencia al medio y la tendencia a contribuir a la formación de jóvenes investigadores.

A partir del año 2011, se realiza el Concurso "Mejor Trabajo presentado por Becarios", destinado a fomentar la participación y visibilidad de sus investigaciones, sometiéndolas al análisis de la comunidad científica.

Las distinciones se entregan a los becarios más destacados por su labor investigadora en cinco áreas del conocimiento: Ciencias Médicas, Ciencias Agropecuarias, Ciencias Exactas y Naturales, Tecnologías, y Ciencias Sociales, Humanísticas y Artísticas.

Dichas jornadas han sido realizadas alternadamente en las ciudades de Resistencia y Corrientes, principales sedes de la Casa de Altos Estudios, de carácter regional. Su vigésima edición se desarrolló entre los días 17 y 19 de junio de 2014 en la Ciudad de Corrientes, con la presentación de más de 300 trabajos desarrollados en el seno de la Universidad.

Tras un proceso de evaluación llevado a cabo por comisiones designadas al efecto, en el área de Ciencias Sociales, Humanísticas y Artísticas, se seleccionaron tres trabajos en la categoría de post-grado y cinco en la de pre-grado, que fueron expuestos en la jornada inaugural.

En la categoría de Becario de Pregrado, el jurado destacó la ponencia 
"Análisis sobre el sistema de valor de la producción sojera en la Provincia del Chaco", presentada por Carlos Harold Gabriel Lucas, alumno de la carrera de Licenciatura en Administración de la Facultad de Ciencias Económicas, otorgándole el primer premio.

En función de tal distinción, el becario expuso parte de los resultados de la investigación sobre "Eficiencia colectiva en la instalación de clúster en la base de la cadena de valor de la producción de soja en la provincia del Chaco", vinculada al proyecto de investigación "Figuras asociativas en la producción de soja en la Provincia del Chaco. Grado de desarrollo de su información contable”, que se desarrolla bajo la dirección de la Licenciada Susana Gusinsky de Gelman y la subdirección de la Contadora Rosa Cruz de Innocente. Particularmente, para este trabajo, el becario contó con la guía de las Contadoras Idalia de Castro y Susana Jerez.

Por su parte, como Becario de Posgrado, categoría iniciación, el Abogado Especialista Lucio Andrés Terrasa, presentó el trabajo titulado "La protección del débil jurídico como meta del derecho posmoderno: paralelismo entre el Derecho del Trabajo y el Derecho de Consumo", realizado en el marco del desarrollo del proyecto de investigación "Funciones actuales del sistema de responsabilidad del proveedor frente al consumidor”, cuya dirección está bajo la responsabilidad de la Dra. María Laura Estigarribia Bieber, con la Codirección del Dr. Cristian Ricardo Piris y la Subdirección de la Abogada Verónica Glibota Landriel, en la Facultad de Ciencias Económicas.

Ambos trabajos fueron galardonados por la UNNE, al ser seleccionados para representarla en las XXII Jornadas de Jóvenes Investigadores de la Asociación de Universidades Grupo Montevideo (AUGM), a realizarse en la Universidad de Playa Ancha, Valparaíso, Chile, los días 29 de septiembre al 1 de octubre de 2014. 